\title{
A systematic approach of tracking and reporting medication errors at a tertiary care university hospital, Karachi, Pakistan
}

\author{
Khurshid Khowaja' \\ Rozmin Nizar' \\ Rashida J Merchant ${ }^{2}$ \\ Jacqueline Dias ${ }^{3}$ \\ Irma Bustamante-Gavino ${ }^{4}$ \\ Amina Malik' \\ 'Division of Nursing Services, \\ ${ }^{2}$ Nursing Education Services, \\ ${ }^{3}$ Diploma Programme, Nurudin \\ Jivraj Professorship of Nursing, Aga \\ Khan University, Karachi, Pakistan; \\ ${ }^{4}$ The Ahmed Shivji Professorship \\ of Nursing, The Aga Khan University \\ School of Nursing, Karachi, Pakistan
}

Correspondence: Khurschid Khowaja Division of Nursing Services, Aga Khan University, PO Box 3500, Karachi 74800, Pakistan

Tel +92 2l 4863600

Fax +92 2I 493 4294/+92 2I 4932095

Email khurshid.khowaja@aku.edu
Introduction: Administering medication is one of the high risk areas for any health professional. It is a multidisciplinary process, which begins with the doctor's prescription, followed by review and provision by a pharmacist, and ends with preparation and administration by a nurse. Several studies have highlighted a high medication incident rate at several healthcare institutions.

Methods: Our study design was exploratory and evaluative and used methodological triangulation. Sample size was of two types. First, a convenient sample of 1000 medication dosages to estimate the medication error $(95 \% \mathrm{CI})$. We took another sample from subjects involved in medication usage processes such as physicians, nurses, pharmacists, and patients. Two sets of instruments were designed via extensive literature review: a medication tracking error form and a focus group interview questionnaire.

Results: Our study findings revealed $100 \%$ compliance with a computerized physician order entry (CPOE) system by physicians, nurses, and pharmacists. The main error rate was $5.5 \%$ and pharmacists contributed an higher error rate of $2.6 \%$ followed by nurses $(1.1 \%)$ and physicians (1\%). Major areas for improvement in error rates were identified: delay in medication delivery, lab results reviewed electronically before prescription, dispension, and administration.

Keywords: medication error rate, associate error rate, physician, nurse, pharmacist

\section{Introduction}

Medication errors are among the most common medical errors, harming at least 1.5 million people every year and adding to US $\$ 3.5$ billion a year in extra hospital costs alone (IOMNA 2006). An estimated 44,000 to 98,000 patients die from medical errors and more than 7,000 deaths occur due to medication errors (IOM 1999). Medication is probably one of the highest risk areas for any health professional (Webster 2001). Besides injuring patients, medication errors cost money and waste time, and also cause loss of life-long productivity in particular pediatric populations (Davis 1995; Buck 1999). Medication usage is a multidisciplinary process, which begins with the doctor's prescription, is followed by the review and provision of medications by a pharmacist, and ends with the preparation and administration of the medication to the patient by a nurse. Inadvertent errors or accidents are encountered if there is a breakdown at any stage in this process, which leads to overwhelming consequences for the patient and for the career of the healthcare professional (Gladstone 1995). The impact of medication errors can be devastating to the confidence and self-esteem of the healthcare professional. Consequently, healthcare professionals are unwilling and indisposed to report any medication error unless there is an obvious harm to the patient. The reluctance comes from apprehension of retribution which could range from corrective actions to termination from work. Healthcare institutions can 
create a culture of medication error reporting by moving from blaming the individual responsible to blaming a system failure.

Several studies have highlighted the medication incident rate at several healthcare institutions (Gladstone 1995; Pape 2001; Webster 2001; Hurley 2006). According to Davis and Drogasch (1997), there is a wide acknowledgment that the number of medication errors is underreported. The reported medication error rate ranges from $1.6 \%$ to $38 \%$ of all medications administered, and it is estimated that only $25 \%$ of errors are reported. According to a study conducted at two academic institutions by Kaushal and colleagues (2001), there were 616 medications errors $(5.7 \%)$ or 55 medication errors per 100 admissions. Flynn and colleagues (2003) reported that "pharmacist confirmed 457 of the 2556 comparison doses to be error, producing a true error rate of 17.9\%". According to Osborne, Blais, and Hayes reported by Pape (2001), approximately $1.6 \%$ to $38 \%$ of all medications administered are in error, excluding approximately $25 \%$ of those not reported.

The Aga Khan University Hospital (AKUH) is an acute tertiary care hospital in Pakistan, with an average daily census of 400 patients at midnight. The hospital's pharmacy data shows that each patient on average receives five medications and 10-12 doses in a 24-hr cycle. The nursing division at AKUH captures 6 to 7 medication errors per month, and compiles an average of 72 to 78 errors annually. The pharmacy department reports 4056 annual potential medication errors which are identified and prevented before actual error occurs. Combining these two reports contributes a $0.7 \%$ medication error rate in 2003. In 2004, medication incidences were 128 and pharmacy reported 5933 potential medication errors giving an error rate of $0.98 \%$ (unpublished data). The current estimates of medication error rate at AKUH are considered low, as many errors are undocumented and unreported. The healthcare providers feel that errors resulting in serious harm are reported because they are easy to identify and hard to conceal, yet they represent the "tip of the iceberg." Reported errors are a small subset of the totality of errors that signal major system breakdowns with possible grave consequences for patients.

Most hospitals have a system of checks and balances to ensure patient safety during medication administration. The issues surrounding medication errors demand a thorough examination of all causes and improvement of medication error systems. A systematic and multidisciplinary approach is, therefore, imperative in order to pick-up errors and devise strategies to reduce harm.

\section{Literature review}

The literature review in this section will highlight definition of medication errors, consequences of underreporting of medication errors followed by risk/contributing factors associated with medication errors.

According to Kaushal and colleagues (2001) medication errors are defined as errors in drug ordering, transcribing, dispensing, administering, or monitoring. Which includes medications: Omitted, given at the wrong time, given to the wrong patient, the wrong dose, the wrong medication, the result of a transcription error, given to a patient with a known allergy, repeated without an order, given by the wrong route, and discontinued without an order (Hartwig et al 1991; Roseman and Booker 1995; Flynn et al 2002; Meadows 2003; Thomsen and Schroeder 2004; Bryony 2003).

Medication errors can be broadly considered to be of three types as described by Barber and colleagues (2003): prescribing errors, dispensing errors, and administration errors. Errors occur most commonly at the stage of drug ordering, dosing errors, and errors involving the intravenous route are the most frequent. What led to these studies are the observations that nearly half of all medication errors occur during the prescribing process with a subsequent cascade effect, which causes errors downstream in dispensing or administration (Thomsen and Schroeder 2004). The most common types of errors include giving the wrong drug or wrong dose, using the wrong route or failure to check the patient's identity (Allan et al 1995; Ferner et al 1999).

\section{Underreporting of medication errors}

Underreporting of medication error is a major challenge for all healthcare facilities and medication errors are often underreported if the provider perceives no harm to the patient or the errors are not considered worthy of reporting (Allan and Barker 1990; Wilson et al 1998). Underreporting of medication errors by nurses is due to fear of reaction from the nurse managers and coworkers, fear of punishment, complex and low self-esteem, organizational factors, and potential termination from job (Beardsley 1999; William 1999; Uribe et al 2002). Ineffective reporting of medication errors occurred in all medication distribution categories: prescribing, transcribing, dispensing, and administration (Hirtz et al 2002).

\section{Risk and contributing factors associated with medication errors}

There are many factors that are associated with drug errors, including inadequate knowledge and skills, failure to comply with policies, and failure in communication. Other 
major contributing factors most likely to be ignored are the complex and poorly designed systems, poor teamwork, and psychological and environmental stressors such as fatigue, anxiety, poor lighting, and noise. The safest work environments address these issues by designing systems to prevent errors, make errors visible, and mitigate the effects of errors (Kaushal et al 2001).

\section{Study goal}

The over all goal of this study was to track medication errors through a systematic approach and explore reasons of nonreporting of medication errors by healthcare professionals.

\section{Implications of study}

The findings of this study will help nursing, physician, pharmacy, and hospital administration and nursing students to launch and implement better strategies to create a culture where healthcare providers feel comfortable in reporting errors and will promote a culture of patient safety and improve health-related outcomes at a tertiary care university hospital in Karachi, Pakistan.

\section{Research objectives}

1. To explore medication error rate through systematic approach.

2. To identify reasons of underreporting of medication errors.

3. To develop and recommend strategies to the hospital management for creating a cultural change where hospital employees feel comfortable in reporting errors.

\section{Research methodology}

An exploratory and evaluative study design, using methodological triangulation, explored the intended purpose of this study. Study was carried out in medical, surgical, obstetrics/ gynecology, and pediatric units.

The sample was of two types. The first convenient sample of 1000 medication dosages was taken to estimate the medication error rate with a $95 \%$ confidence interval (CI). Therefore, to estimate medication error rate in this study, with a bound on error of $0.05(5 \%)$ with a $95 \%$ CI needed to take at least $n \geq \frac{Z(\alpha / 2)^{2} p q}{B^{2}}$, the maximum sample size was computed to be 1000 dosages when $\mathrm{p}$ was taken as 0.7 .

The other sample set was from the subjects involved in medication usage processes such as physicians, nurses, pharmacists, and patients. Focus group interviews were conducted with two groups of registered nurses (each group included 8-10 nurses from selected units), one group of doctors (8-10 physicians), one group of pharmacists (8-10 pharmacists), and two groups of patients (16-20 patients).

\section{Operational definition of medication error}

The authors have defined medication error in this study by using the definition by the National Coordinating Council for Medication Error Reporting and Prevention: "A medication error is any preventable event that may cause or lead to inappropriate medication use or patient harm while the medication is in the control of the health care professional, patient, or consumer. Such events may be related to professional practice, health care products, procedures, and systems, including prescribing; order communication; product labeling, packaging, and nomenclature; compounding; dispensing; distribution; administration; education; monitoring; and use" (NCC MERP 2008).

\section{Ethical considerations}

Permission was obtained from the Ethical Review Committee of the tertiary care university hospital at Karachi, Pakistan. Written permission was obtained from study subjects (patients) as this was an observational study, and study subjects were kept blind to prevent information biases as capture of accurate error rates would be difficult if they were aware. Confidentiality of data was maintained by assigning special codes to study subjects. Written consent was obtained from the subjects of focus group interview prior conducting the interviews. The authors decided that if the data collectors observed any moderate injury (injury in which length of hospital stay did not increase, but required some treatment or intervention) or severe medication injury (injury was serious, caused considerable discomfort to patients, and required extended treatment or was life-threatening) he/she would immediately inform the head nurse of the unit to ensure patient safety.

\section{Inclusion and exclusion criteria}

- 1000 medication dosages from patients staying 3-4 days in the selected units (B1, PWI, C1, C2, PWII, B2, and Peads A) included in the sample.

- Those registered nurses, physicians, pharmacists with one year or more experience included in the sample. However, patients for focus group interview were selected from these who had a length of stay of 3-4 days.

\section{Study instruments}

Two sets of data instruments were designed via extensive literature review to capture all types of medication errors. 
1. Tracking of medication error form. This questionnaire is very comprehensive and meets the requirement for fulfilling study objectives (Kozier et al 1995).

2. Focus group interview questionnaire.

\section{Design of study instruments}

Both study instruments were designed in a simple language that can be easily understood by data collectors and study participants. The first instrument was developed for English language speakers and contained all components of medication usage. The focus group interview questionnaire for healthcare professionals was also designed in English. However it was translated in Urdu for patients who were not able to understand English. To ensure accuracy in English translation, the same Urdu questionnaire was translated back into English

\section{Data collection process}

A data collector was hired and trained to understand the complexities of the physician order entry (POE) system and medication administration for medication error rate tracking. $\mathrm{He} / \mathrm{she}$ collected the data from all 3 shifts including morning, evening, and night. Data collection was done through multiple approaches to cover all aspects of the medication system. The principal investigator and co-investigators ensured the quality of data collection by random visits to the data collection sites in order to check whether the questionnaires were completed properly or not. Moreover, the questionnaire was edited twice for missing information or incorrect responses in the field and again at the office.

\section{Statistical analysis}

Descriptive analysis was performed to describe data for frequency, mean, and standard deviations for continuous variables: sociodemographic variables and proportions calculated in categorical variables. Descriptive analysis was also done to calculate the medication error rate. Further analysis will be to correlate different data variables to assess the areas of magnitude contributing factors. The focus group interview will highlight themes and areas of importance for creating a culture for medication error reporting.

\section{Results}

Data was collected from all three shifts: morning, evening, and night. The sample was almost equally distributed among all 3 shifts: ie, 300 dosages (30\%) from the morning sample, 350 dosages (35\%) from the evening sample, and 350 dosages $(35 \%)$ from the night sample. Sample was selected from all medical/surgical and one pediatric units. Study findings revealed $100 \%$ compliance with the POE system by physicians, nurses, and pharmacists. The main error rate was $5.5 \%$. Pharmacy contributed a higher error rate of $2.6 \%$, followed by nursing $1.1 \%$, and physicians $1 \%$ as indicated in Figure 1. In the nurse error rate, a major area of consideration was the wrong route: 7 medication dosages were administered via the wrong route, four dosages given to the wrong patient, four were incorrect drugs administered, and four incorrect doses. In the pharmacy error rate, 22 were wrong dosages, and in the physician error rate, six medication routes were incorrectly written.

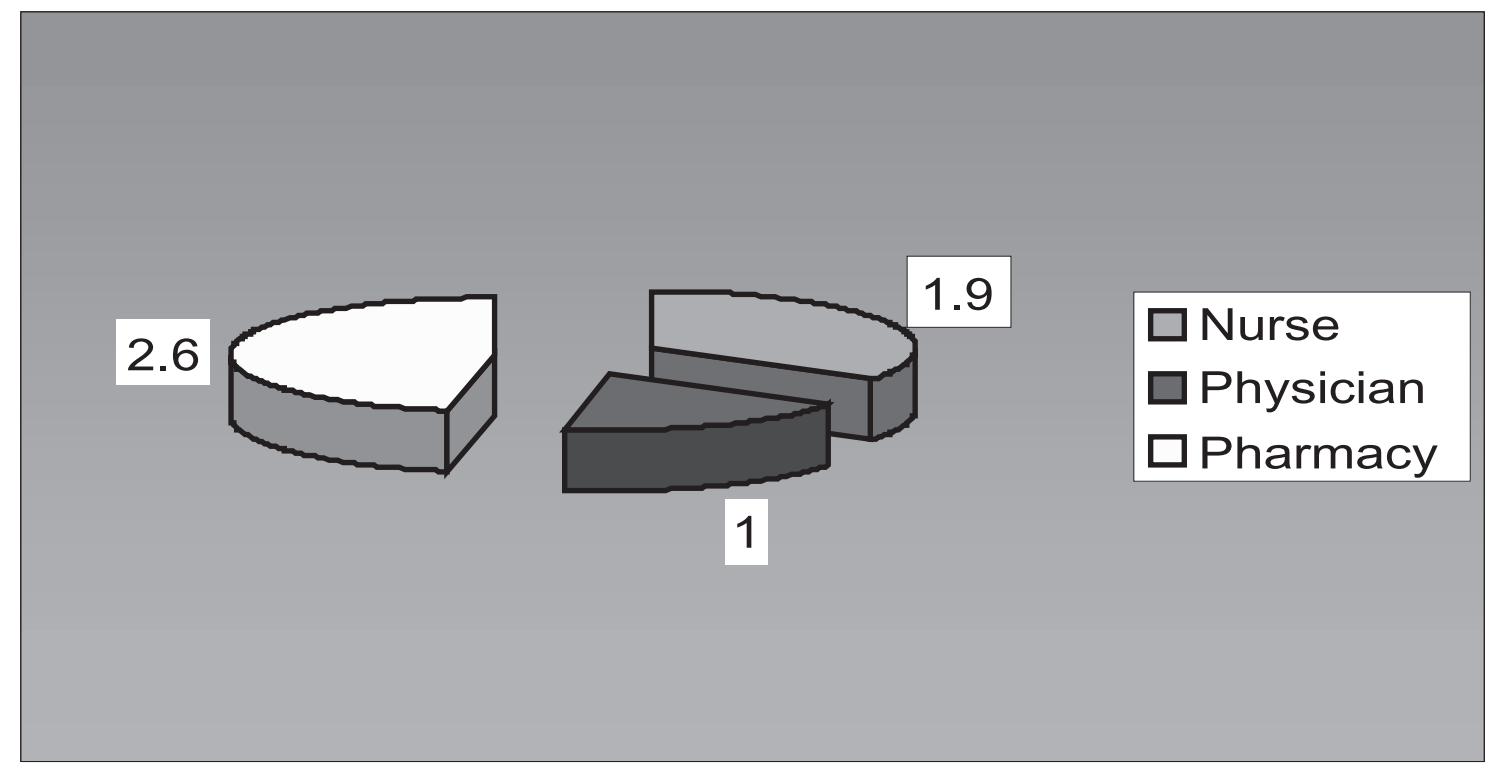

Figure I Main error rate 5.5\%. 
The associate error rate was categorized by two areas: right time and review of lab values before prescription. The right time area showed an associate error rate of $26.4 \%$. In this area, pharmacy contributed a high error rate of 193 $(19.3 \%)$ dosages not delivered to patient care areas on time and therefore were administered late by nursing staff. As per policy on medication usage, all stat medications are administered in 30 minutes and routine in 45 minutes. Furthermore, the review of lab values before prescription as indicated in Figure 2 depended on correct dispending and administration. Data revealed that 690 (69\%) lab values of dosage were not electronically reviewed by physicians, 350 (35\%) by nurses, and 350 (35\%) by pharmacists before prescription, dispending, and administration of medication. Data also identified in knowledge regarding medication usage among all three providers (physicians, nurses, and pharmacists), that physicians contributed higher knowledge regarding dosage, indication, and side effects in comparison with nurses and pharmacists. However, all three providers' knowledge regarding medication compatibility was much less. The pharmacist had 750 (75\%) knowledge regarding dosage's compatibility, whereas physician knowledge was $520(52 \%)$, and nurse knowledge was only $130(13 \%)$. Nurses also had less knowledge regarding indication, side effects, and other areas of medication usage.

To discover the reasons for underreporting of medication errors, focus group interviews were conducted from registered nurses, physicians, and pharmacists. Sixteen nurses,
8 physicians, 8 pharmacists, and 16 patients participated in the focus group interview. Physicians and nurses responded that the main reason for occurrences of errors was high workload, lack of POE knowledge, and poor time management. All healthcare professionals agreed that they were aware of the process of error reporting on the yellow incident and yellow ADR forms. They also verbalized that all errors were not reported, in particular physician-related prescription errors as they were either picked up by pharmacy and nursing departments before they reached patients, and physicians were paged to correct or confirm their orders. Study participants suggested that adherence can drastically reduce error rate and they further suggested that workload on frontline physicians and nurses should be reduced and a supportive management style can create a culture of timely error reporting. Furthermore, they added that nursing knowledge regarding medications should be enhanced via tutorials.

\section{Discussion}

Important findings in this study was that the main error rate was $5.5 \%$ and pharmacy contributed a higher error rate of $2.6 \%$, followed by nursing $1.1 \%$ and physicians $1 \%$. The computerized POE system at AKUH provided a reduced error rate by physicians. Focus group interviews identified that the main reasons for underreporting of medication errors were fear of disciplinary process and loss of job. Pepper and Chiang (2006) reported similar findings and identified fear and administrative barriers as the top two reasons for

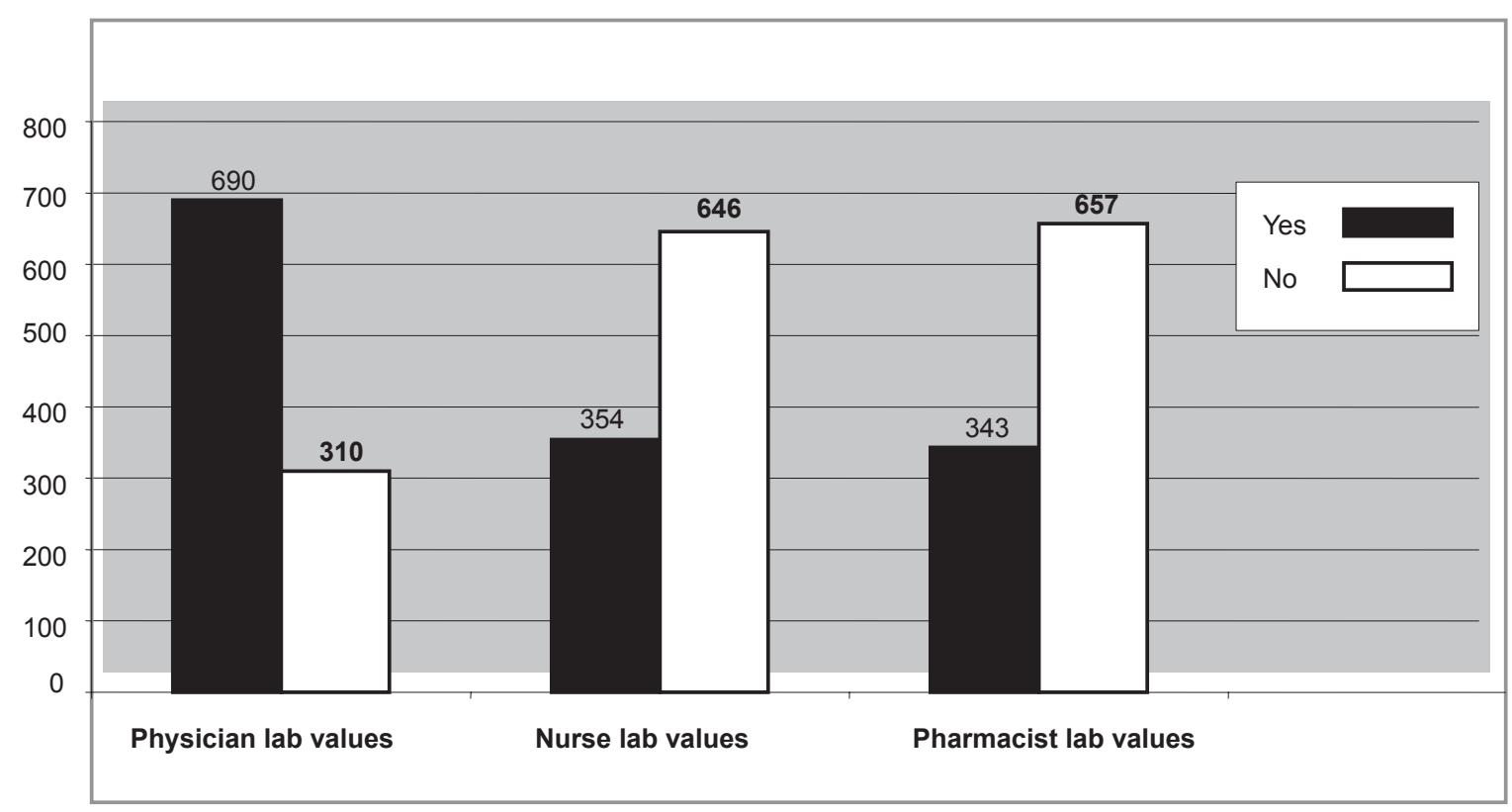

Figure 2 Review of lab values. 
underreporting. The focus group interviews with patients and staff revealed the following themes: appropriate staffing, decreased workload, increased communication among team members, more emphasis on knowledge enhancement, in particular POE and drug compatibility, and a supportive work environment to prevent loss of job for reporting medication errors.

\section{Limitations}

The reasons for late medication delivery at the pharmacy could be due to clarification of physician orders by clinical pharmacists.

\section{Recommendations}

Our study findings generated the following recommendations for tracking and reducing medication errors:

- The computerized POE system is very useful in inpatient as well as in ambulatory units along with bar coding and electronic medication administration records input by nurses. This system not only detects and prevents errors but prevents delays at all level of prescription, administration, and dispension. However, POE presents several possible dangers by introducing new types of errors such as slower order entry by prescribers and inexperienced staff, utilized more staff time, slower person-to-person communication in an emergency situation, lack of communication between physicians and nurses, automation caused a false sense of security, shortcut or default selections can override nonstandard medication regimens for elderly or underweight patients, which resulted in toxic doses, and frequent alerts and warnings can interrupt work flow (Koppel et al 2005; Lohr 2005).

- All medication received by nursing units should be tagged with the time determine delays in delivery as the associated error rate was $26.4 \%$ in our current study due to delayed delivery by pharmacists and delayed administration by nurses. Tracking of medication errors from verification to dispending, delivery, and administration should be electronic-based to prevent error occurrences.

- Post-Graduate Medical Education (PGME), School of Nursing (SON), and Nursing Education Services (NES) should teach nurses and physicians more about drug compatibility.

- Comprehensive multidisciplinary medication usage polices and audit tools should be designed and compliance measurements should be done every month to ensure patient safety.

- In considering medication usage as a high risk area and prime safety goal, tracking errors should be an indicator for all nursing units. Clinical practice and nursing management should do point prevalence and periodic reviews every month to ensure patient safety.

- Plan Do Study and Act (PDSA) projects/quality circles should be launched in nursing units with high medication error rates.

- Lab values should be reviewed by pharmacists before dispending drugs.

- Install software for tracking medication errors.

- The NCC MERP error classification index should be used at AKUH to classify errors according to severity (Figure 3 ).

\begin{tabular}{|c|c|c|}
\hline \multicolumn{3}{|c|}{ Medication Error Index ${ }^{1}$ : } \\
\hline Type & Category & Result \\
\hline No Error & Category A & Circumstances or events that have the capacity to cause error. \\
\hline \multirow{3}{*}{$\begin{array}{l}\text { Error, No } \\
\text { Harm }\end{array}$} & Category B & An error occurred, but the medication did not reach the patient. \\
\hline & Category C & An error occurred that reached the patient, but did not cause the patient harm \\
\hline & Category D & $\begin{array}{l}\text { An error occurred that resulted in the need for increased patient monitoring, but not } \\
\text { patient harm. }\end{array}$ \\
\hline \multirow[t]{4}{*}{ Error, Harm } & Category E & $\begin{array}{l}\text { An error occurred that resulted in the need for treatment or intervention and caused } \\
\text { temporary patient harm. }\end{array}$ \\
\hline & Category F & $\begin{array}{l}\text { An error occurred that resulted in initial or prolonged hospitalization and caused } \\
\text { temporary patient harm. }\end{array}$ \\
\hline & Category $\mathrm{G}$ & An error occurred that resulted in permanent patient harm. \\
\hline & Category $\mathrm{H}$ & $\begin{array}{l}\text { An error occurred that resulted in a near-death event (e.g., anaphylaxis, cardiac } \\
\text { arrest). }\end{array}$ \\
\hline Error, Death & Category I & An error occurred that resulted in patient death. \\
\hline
\end{tabular}

Figure 3 Medication error index (NCC MERP 2008). 


\section{Conclusion}

The patient-centered approach and supportive culture will encourage healthcare professionals to report medication errors. Implementation of error reduction strategies, more point prevalence as per medication usage tools, on-going incident reporting schemes, and rewards to those who report errors, categorization of medication errors as per a severity index, and use of software programs for medication tracking will allow a reduced error rate and promote patient safety.

\section{Disclosure}

The authors report no conflicts of interest.

\section{References}

Allan EL, Barker KN, Malloy MJ, et al. 1995. Dispensing errors and counseling in community practice. Am Pharm, NS35:25-32.

Allan EL, Barker KN. 1990. Fundamentals of medication error research. Am J Hosp Pharm, 47:555-71.

Barber N, Rawlins M, Franklin BD. 2003. Reducing prescribing error: competence, control and culture. Qual Saf Health Care, 12:129-32.

Beardsley D. 1999. Board of nursing decision puts patients at risk. J Nurs Admin, 29:1-2.

Buck ML. 1999. Preventing medication errors in children. Pediatric Pharmacology. A Monthly Review for Health Care Professionals of the Children's Medical Centre. 5(10):1-8.

Bryony DF. 2003. Medication errors - what's the story? FIP Hospital Pharmacy Section Newsletter, 5:3.

Clinical management intravenous medication administration [online]. 2008. Accessed on 5 February, 2008. URL: http://www.darlingtonpct.nhs. uk/documents.

Davis JT. 1995. Clinical pathway can be based on acuity not on diagnosis. Ann Thoracic Surg, 59:1074-8.

Davis L, Drogasch M. 1997. Triple check procedure prevents chemotherapy errors. Oncol Nurs Forum, 24:641-2.

Ferner RE, Aronson JK.1999. Errors in Prescribing, preparing and giving medicines: definition, classification and prevention [online]. Accessed on May 10, 2008. URL: www.hero.org.ph/files/pharmacovigilance.pdf.

Flynn EA, Barker KN, Pepper GA, et al. 2002. Comparison of methods for detecting medication errors in 36 hospitals and skilled-nursing facilities. Am J Health Syst Pharm, 59:436-46.

Flynn EA, Barker KN, Carnahan BJ. 2003. National observational study of prescription dispensing accuracy and safety in 50 pharmacies. $\mathrm{J} \mathrm{Am}$ Pharm Assoc, 43:191-200.

Gladstone J. 1995. Drug administration errors: a study into the factors underlying the occurrence and reporting of drug errors in a district general hospital. J Adv Nurs, 22:628-37.
Hartwig SC, Denger SD, Schneider PJ. 1991. Severity-indexed, incident report-based medication error-reporting program. Am Health Syst Pharm, 48:2611-16.

Hirtz RW, Everly JL, Sandra A. 2002. Medication error identification is a key to prevention: A performance improvement approach. $J$ Healthc Qual, 24:10-17.

Hurley AC, Lancaster D, Hayes J, et al. 2006. The Medication Administration System-Nurses Assessment of Satisfaction (MAS-NAS) Scale. J Nurs Scholarsh, 38:298-300.

[IOM] Institute of Medicine; Kohn LT, Corrigan JM, Donaldson MS (eds) 1999. To Err is Human: building a safer health system [online]. Accessed on February 5, 2008. URL: http://www.iom.edu/File.aspx?ID=4117.

[IOMNA] Institute of Medicine of the National Academies. 2006. Preventing medication errors. Quality Chasm series. Washington, DC: National Academies Press, July 2006.

Kaushal R, Bates DW, Landrigan C, et al. 2001. Medication errors and adverse drug events in pediatric inpatients. JAMA, 285:2114-20.

Kozier B, Erb G, Blais K, et al. 1995. Fundamentals of Nursing: Concepts, Process and Practice. 5th ed. New York, NY: Addison Wesley Nursing.

Lohr S. 2005. Doctors' Journal Says Computing Is No Panacea [online]. Accessed on October 15, 2006. The New York Times, 2005-03-09.

Meadows M. 2003. Strategies to reduce medication errors. FDA Consumer Magzine, 2003 [online]. Accessed on February 5, 2008. URL: http:// www.fda.gov/fdac/feaures/2003/303_meds.html.

[NCC MERP] National Coordinating Council for Medication Error Reporting and Prevention. 2008. National Coordinating Council for Medication Error Reporting and Prevention. 1998-2006 [online]. Accessed on February 5, 2008. URL: http://www.nccmerp.org/councilRecs.html.

Pape TM. 2001. Searching for the final answer: Factors contributing to medication administration errors. J Contin Educ Nurs, 32:152-60.

Pepper GA, Chiang HY. 2006. Barriers to nurses' reporting of medication administration errors in Taiwan. J Nurs Scholarsh, 38:392-9.

Koppel R, Metlay JP, Cohen A, et al. 2005. Role of computerized physician order entry systems in facilitating medication errors. JAMA, 293:1197-203.

Roseman C, Booker JM. 1995. Workload and environmental factors in hospital medication errors. J Nurs Admin, 44:226-30.

Thomsen CJ, Schroeder RW. 2004. The scope of medication error problem [online]. Accessed on February 5, 2008. URL: http://www.touchbriefings.com/pdf/790/30-thomsen.pdf.

Webster CS. 2001. A systems approach to the reduction of medication error on the hospital ward. J Adv Nurs, 35:34-41.

William GW. 1999. Board of nursing decision puts patients at risk. J Nurs Admin, 29:4-5.

Wilson RG, McArtney RG, Newcombe RJ, et al. 1998. Medication errors in paediatric practice: insights from a continuous quality improvement approach. Eur J Pediatr, 157:769-74.

Uribe CL, Schweikhart SB, Pathak DS, et al. 2002. Perceived barriers to medical error reporting; An Exploratory investigation. J Health Care Manage, 47:263-80. 
